\title{
Report on plasma jet printer for sensor fabrication with process parameters optimized by simulation input
}

Mike McMurtrey

Kunal Mondal

Kiyo Fujimoto

Joseph L. Bass

Austin Biaggne

September 2019

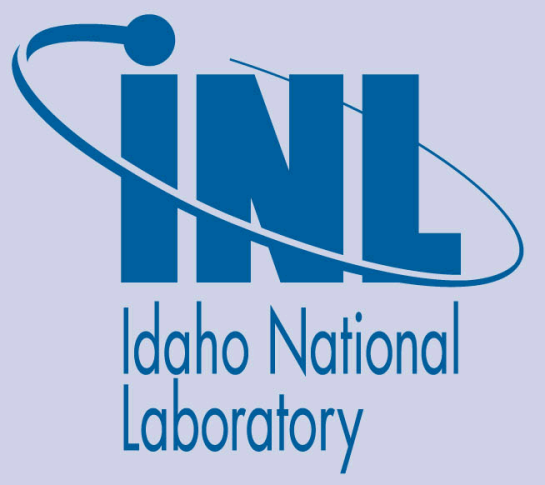




\section{DISCLAIMER}

This information was prepared as an account of work sponsored by an agency of the U.S. Government. Neither the U.S. Government nor any agency thereof, nor any of their employees, makes any warranty, expressed or implied, or assumes any legal liability or responsibility for the accuracy, completeness, or usefulness, of any information, apparatus, product, or process disclosed, or represents that its use would not infringe privately owned rights. References herein to any specific commercial product, process, or service by trade name, trade mark, manufacturer, or otherwise, does not necessarily constitute or imply its endorsement, recommendation, or favoring by the U.S. Government or any agency thereof. The views and opinions of authors expressed herein do not necessarily state or reflect those of the U.S. Government or any agency thereof. 
INL/EXT-19-55831

Revision 0

\title{
Report on plasma jet printer sensor fabrication with process parameters optimized by simulation input
}

\author{
Mike McMurtrey ${ }^{1}$ \\ Kunal Mondal ${ }^{1}$ \\ Kiyo Fujimoto ${ }^{1,2}$ \\ Joseph L. Bass ${ }^{1}$ \\ Austin Biaggne $^{2}$
}

September 2019

${ }^{1}$ Idaho National Laboratory Idaho Falls, Idaho 83415

${ }^{2}$ Boise State University

Boise, Idaho 83725

http://www.inl.gov

Prepared for the

U.S. Department of Energy

Office of Nuclear Energy

Under DOE Idaho Operations Office

Contract DE-AC07-05ID14517 



\begin{abstract}
The In-Pile Instrumentation program conducts research to develop instrumentation that provides information regarding test conditions, as well as fuel and materials properties during irradiation. Such instruments must be miniaturized, robust to the nuclear reactor environment, and provide real-time data aligned with user needs. Towards this end, additive manufacturing techniques such as aerosol jet printing and Plasma Jet Printing, have demonstrated significant potential for the development of advanced in-pile instrumentation. Process control during the manufacturing process is critical in order to consistently create reliable sensors. This report details experimental and computational work aimed towards improved process control for robust sensor production using printing techniques.
\end{abstract}




\section{CONTENTS}

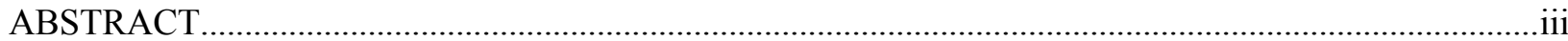

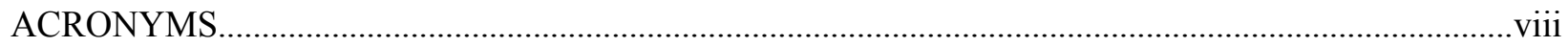

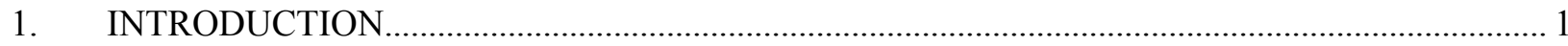

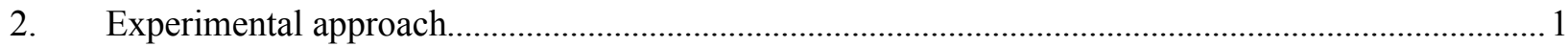

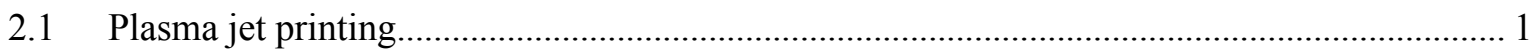

2.2 Advanced printings for melt wire applications............................................................... 3

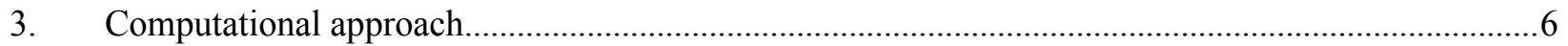

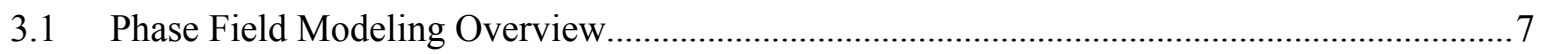

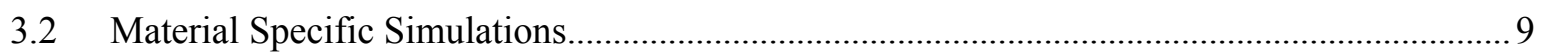

3.2.1 Single Particle Systems............................................................................. 9

3.2.2 Multi-Particle Simulations.......................................................................... 10

4. Nanoparticle (NP) sintering: A density functional theory (DFT) approach................................... 11

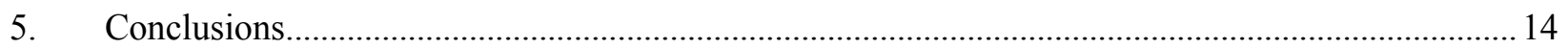

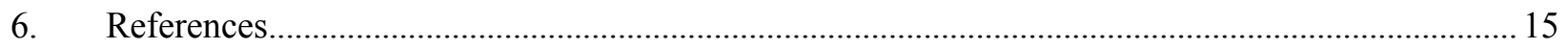

\section{FIGURES}

Figure 1. (a) Digital image of the plasma jet printer and the print head with glowing plasma. (b) Schematic describes the working mechanism of PJP.

Figure 2. (a) Digital image of a PJP printed pattern on a glass substrate, and (b) optical micrograph shows the detailed morphology of printed line. 3

Figure 3. Schematic illustrating the schematic for the melt wire printing and encapsulation.

Figure 4. Printing of melt wires. (a) A blank alumina wafer as a model test substrate, (b) printed melt wires with platinum nanoparticle ink. The inset showing the stock platinum ink solution prior to printing. (c) copper nanoparticle ink as melt wires, and (d) micromachined copper melt wires to fit in the $2 \mathrm{~mm}$-diameter test

reactor modules. The inset shows the optical micrograph of the printed wire. .5

Figure 5. Characterization of printed melt wires. (a) Laser microscopic image of a copper melt wire. The inset shows 3D topography of the wire to estimate width and height of the wire. (b) X-ray computer tomography (CT scan) of the melt wires to confirm that the line will be visible during and after exposure.

Figure 6. Detached printed Ni sensor. 7

Figure 7. TEM Image of nanoparticles 8

Figure 8. (a) Initial vapor phase concentration, (b) equilibrium vapor phase concentration, (c) Initial particle phase concentration, (d) equilibrium particle phase 
concentration, (e) Initial substrate phase concentration, (f) equilibrium substrate phase concentration.

Figure 9. Initial (a) and equilibrium (b) vapor phase concentration for simulation of nickel on alumina substrate at $1000{ }^{\circ} \mathrm{C}$

Figure 10. Initial(a) and equilibrium(b) vapor phase concentration for sintered nickel particles on alumina substrate at $1000{ }^{\circ} \mathrm{C}$

Figure 11. Initial (a) and equilibrium (b) vapor phase concentration for sintered $\mathrm{Al}(\mathrm{left})$ and $\mathrm{Ag}$ (right) particles on alumina.

Figure 12. Sintering of two $1 \mathrm{~nm}$ nanoparticles (one Mo and one $\mathrm{Nb}$ ) using ab-initio molecular dynamics from $0 \mathrm{~K}-800 \mathrm{~K}$.

Figure 13. (a) Plots of surface energy vs. number of atomic layers for various Al slabs of different sizes. (b) Al slab with 6 atomic layers.

Figure 14. Interface of Mo and Alumina made without necessary interface-building software.

\section{TABLES}

Table 1. Literature value for Al surface energy compared to my calculations. [8] 13 


\section{ACRONYMS}

$\begin{array}{ll}\text { AJP } & \text { Aerosol Jet Printing } \\ \text { AM } & \text { Advanced Manufacturing } \\ \text { NP } & \text { Nanoparticles } \\ \text { PJP } & \text { Plasma Jet Printing } \\ \text { X-CT } & \text { X-ray Computed Tomography }\end{array}$




\title{
Report on plasma jet printer sensor fabrication with process parameters optimized by simulation input
}

\author{
1. INTRODUCTION
}

The In-Pile Instrumentation program conducts research needed to develop and deploy unique instruments to characterize the behavior of fuels and materials during irradiation tests conducted by Nuclear Energy programs within the Department of Energy. The vision of this program is to provide real-time, accurate, spatially resolved information regarding performance of fuels and materials during irradiation experiments in nuclear test reactors.

Within the In-Pile Instrumentation program, there are several thrusts dealing with novel sensor designs and cross-cutting activities such as advanced manufacturing (AM) techniques for sensor production. The vision of the AM thrust is to develop a diverse set of AM methods, equipment, and expertise to enable versatile sensor fabrication processes aligned with the five R\&D activities of the initiative. It is anticipated that AM will significantly accelerate the feedback loop of design, modeling, fabrication, and testing by reducing the traditionally time-consuming manufacturing and prototyping time frame, thus enabling rapid advancements for in-pile instrumentation.

Ink jetting techniques, such as aerosol jet printing (AJP) and plasma jet printing (PJP), have been identified as methods that merit development in the area of sensor production due to their flexibility in printing a variety of geometries and materials. However, both AJP and PJP have need for improved process control to ensure the consistent robustness and reliability of the printed sensors. Given the use of the sensors in nuclear test reactors, failures during the experiments can result in costly loss of experimental data. Both experimental, as well as modelling/simulation approaches to improved process control (and sensor reliability) have been examined and will be included in this report.

\section{Experimental approach}

INL has recently acquired PJP capabilities. This is a new technique within the material jetting methods, and there is significant work that needs to be done to ensure accurate and consistent sensor production using PJP. However, there are benefits to this new technique as well, such as the ability to print with improved adhesion to the substrate. This will be discussed further in the following section which will cover a detailed discussion of PJP, as well as preliminary results printing with the PJP.

\subsection{Plasma jet printing}

AJP, a more traditional ink jetting technique, needs post-deposition thermal treatment for the creation of a uniform film and removing residual organic impurities. This post treatment phase leads to void and crack formation in the printed patterns, which affects the quality of the deposited coating and consequence in poor device performance. PJP uses atmospheric dielectric barrier discharge and this acts as a solution to address and overcome the above limitations of AJP.[1] Gandhiraman et al.[2] demonstrated first the idea of an aerosol-assisted atmospheric pressure plasma-based printing technique for depositing ink materials on preferred flexible substrates. This jet printing method is a scalable solution for high-throughput processing and capable of patterning onto 3D complex objects. The plasma serves to drive off the ink fluid, as well as sinter the particles during deposition so there is no need for a high temperature furnace sintering after the printing (as is required by AJP). The other advantage of PJP is that there is no need for vacuum pumps and vacuum chambers since the process takes place at atmospheric pressure. Additionally, the atmospheric pressure plasma performs sterilization and 
plasma treatment of surfaces during the printing process as well, which removes biological contaminations and enhances adhesion between the printed ink patterns and the substrate.

PJP can print a wide range of materials including organics, metals and others. While in-situ plasma sintering, as is performed in the case of PJP, is relatively new, ex-situ plasma sintering has been used on AJP printed inks in place of the typical furnace sintering. Recently, Dey et al.[3] used PJP technique for the in-situ reduction of highly acidic graphene oxide ink. They demonstrated that an atmospheric pressure PJP with $\mathrm{He}$ and $\mathrm{H} 2$ plasma can pattern graphene oxide from a highly acidic precursor and reduce it concurrently. They have extended the printing process further on the printed ink with plasma to enhance the properties of the patterns. The role of plasma here contains sintering and activation of the material present in the printed aerosol ink, enhancing the rate of deposition, and plasma polymerization if inorganic coatings are printed. Tsumaki et al.[4] demonstrated plasma-assisted inkjet printing for direct writing of conductive silver line on a polyimide substrate, where inks were treated with plasma during and after deposition and more importantly, there was no need for any pre-/post-treatments beyond the plasma sintering.

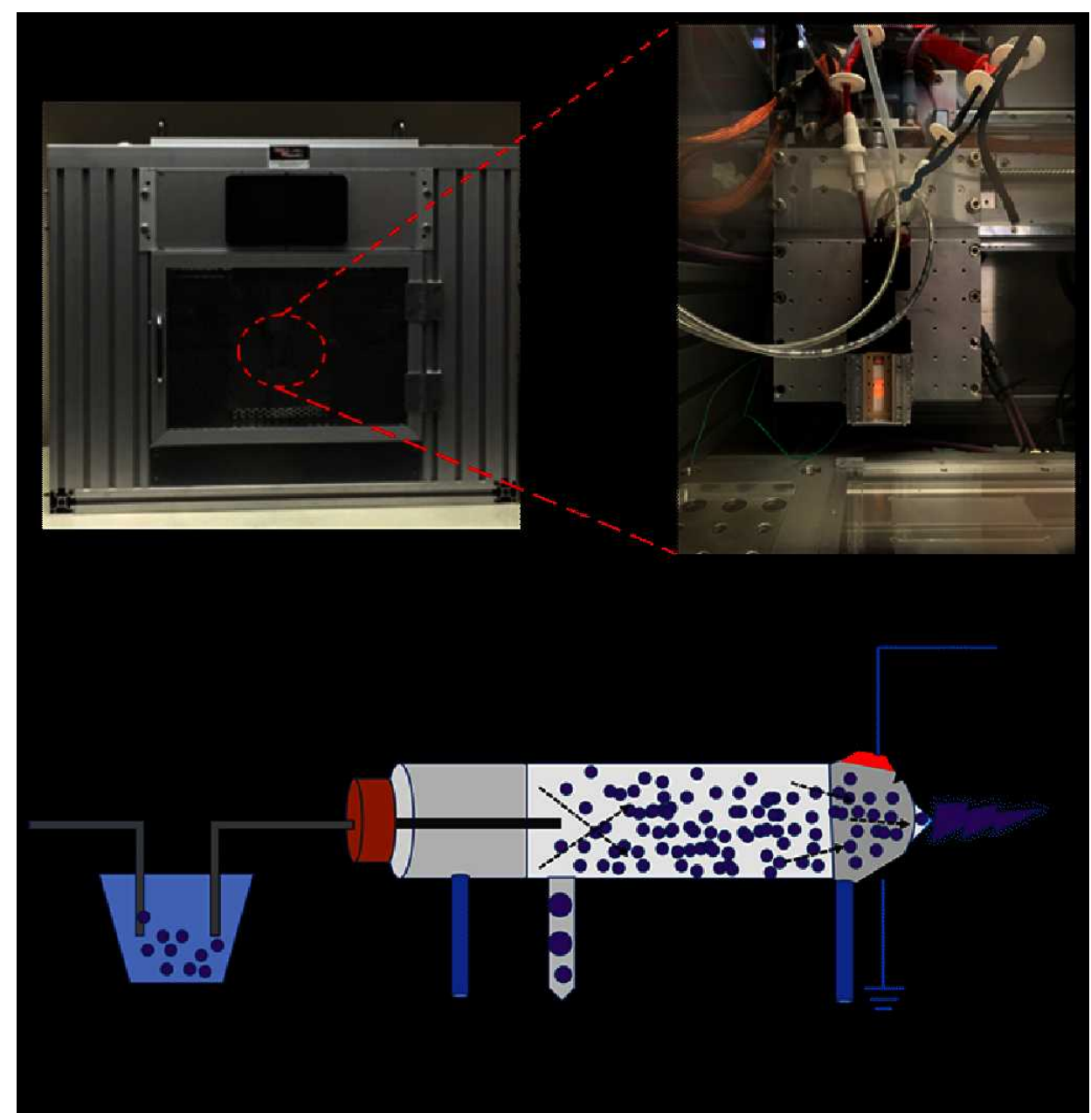

Figure 1. (a) Digital image of the plasma jet printer and the print head with glowing plasma. (b) Schematic describes the working mechanism of PJP.

The plasma jet printer comprises of a quartz nozzle containing two conductive copper 
electrodes ( 1-2 cm spacing) and associated with a high-voltage ( tens of kilovolts) AC power

supply. Fig. 1a shows a digital image of the plasma jet printer along with the zoomed view of the print head. Fig. 1b shows a schematic to describe the of the operation mechanism of plasma jet printing system. A dielectric barrier discharge of inert gas (helium, argon etc.) is created using the high voltage between the electrodes. A bottle containing the colloid of the ink material is located on a nebulizer that produces an aerosol jet of the colloid, and the inert gas carries the aerosol into the quartz tube comprising the plasma. The ink is then deposited on the substrate (nonconductive) placed closely to the nozzle along with the plasma. The printing takes place at room temperature to $50^{\circ} \mathrm{C}$ and for this reason this method termed as cold plasma deposition.

Printing in this program has started with an ink made from copper nanoparticles and the parameters were optimized by observing the resulting printed lines. The major printing parameters optimized were the applied voltage, frequency, flow rate of argon gas, and the zheight of the print head from the substrate. Apart from those above-mentioned options, there are other parameters too which influence the printing and those are the adherence energy of the ink to substrate, the surrounding humidity, and the rheology and chemistry of the ink. The printing was attempted on a glass slides and can be seen in Fig. 2a. The detailed micrograph of the printed copper nanoparticle line of $146 \mu \mathrm{m}$ width is shown in Fig. 2b. A clean plasma treated glass slide was used as substrate. The plasma treatment was performed in order to modify the surface of the glass substrate. The surfaces of glass are treated by plasma to change a hydrophobic/partially hydrophobic surface to hydrophilic by oxidation. Other known results of plasma treatment include dehydrogenation, the formation of radicals, enhance of adhesion energy, and roughening of the surface.[5]

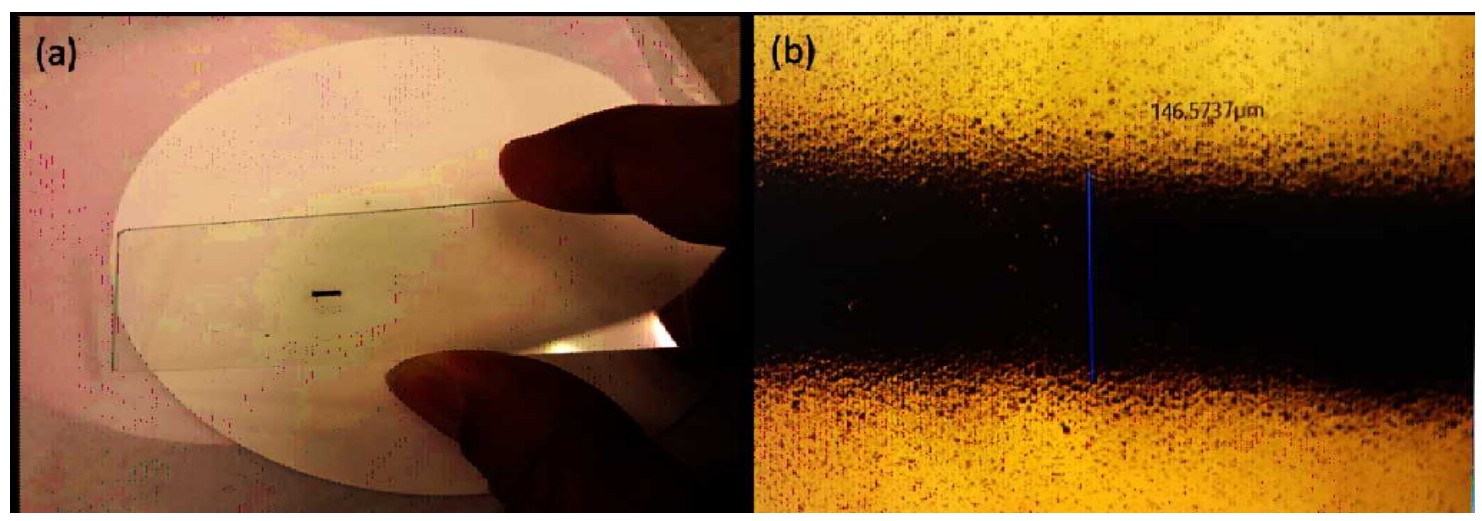

Figure 2. (a) Digital image of a PJP printed pattern on a glass substrate, and (b) optical micrograph shows the detailed morphology of printed line.

\subsection{Advanced printings for melt wire applications}

One of the first applications of PJP will be making a meltwire chip that can be included in geometrically small experiments (such as testing fuel pellets). Melt wires are a passive technique to monitors temperature when real-time sensors are not practical or economical to install in an irradiation test. Melt wires are mainly used for peak temperature evaluation. Even though melt wires have been used at Advanced test reactors (ATR), recent efforts have extended the types offered to users, permitting more precise estimates of peak temperature, 
and improved resulotion, as well as a small chip that is fully encapsulated.

Metal wires of a known material composition and melting temperature are positioned in an irradiation test field to investigate if a definite peak temperature is touched. A post-test radiation inspection of the wire is a must to check if melting happened indicate the corresponding melting temperature was attended or surpassed the melting temperature. Here the PJP will be used to develop in-house capabilities to confirm the melting temperature of selected wire materials (ranging from $\sim 200$ to $1000{ }^{\circ} \mathrm{C}$ ) and to cover multiple melt wires into a single small diameter unit for irradiation testing. Fig. 3 shows a schematic with a circular wafer ( $2 \mathrm{~mm}$ diameter) containing multiple melt wires. The substrate was first micromachined (or etched) to create pockets for the melt wires. Then the printing is performed into the pockets and then encapsulate with another, flat (un-etched) substrate acting as the lid. Examples of meltwires printed on to an alumina substrate can be seen in Fig. $\mathbf{4}$ a-d, with topography shown in Fig. 5a.

Since these wires will be encapsulated, post-test examination is more challenging than typical melt wire visual inspection. X-ray computer tomography (X-CT) has been selected as a method for examining the melt-wires post irradiation. The printed wire on an alumina substrate was examined using X-CT (can be seen in Fig. 5b) to establish the methodology for the precise estimation of melt wires. Fig. $\mathbf{5 b}$ The CT scan shows the melt wires are completely visible to $\mathrm{x}$ rays. The next goal is to use X-CT to check the wires printed and fully encapsulated in a stainless steel substrate, since alumina is not a likely candidate for a melt-wire chip that will go in a test reactor.

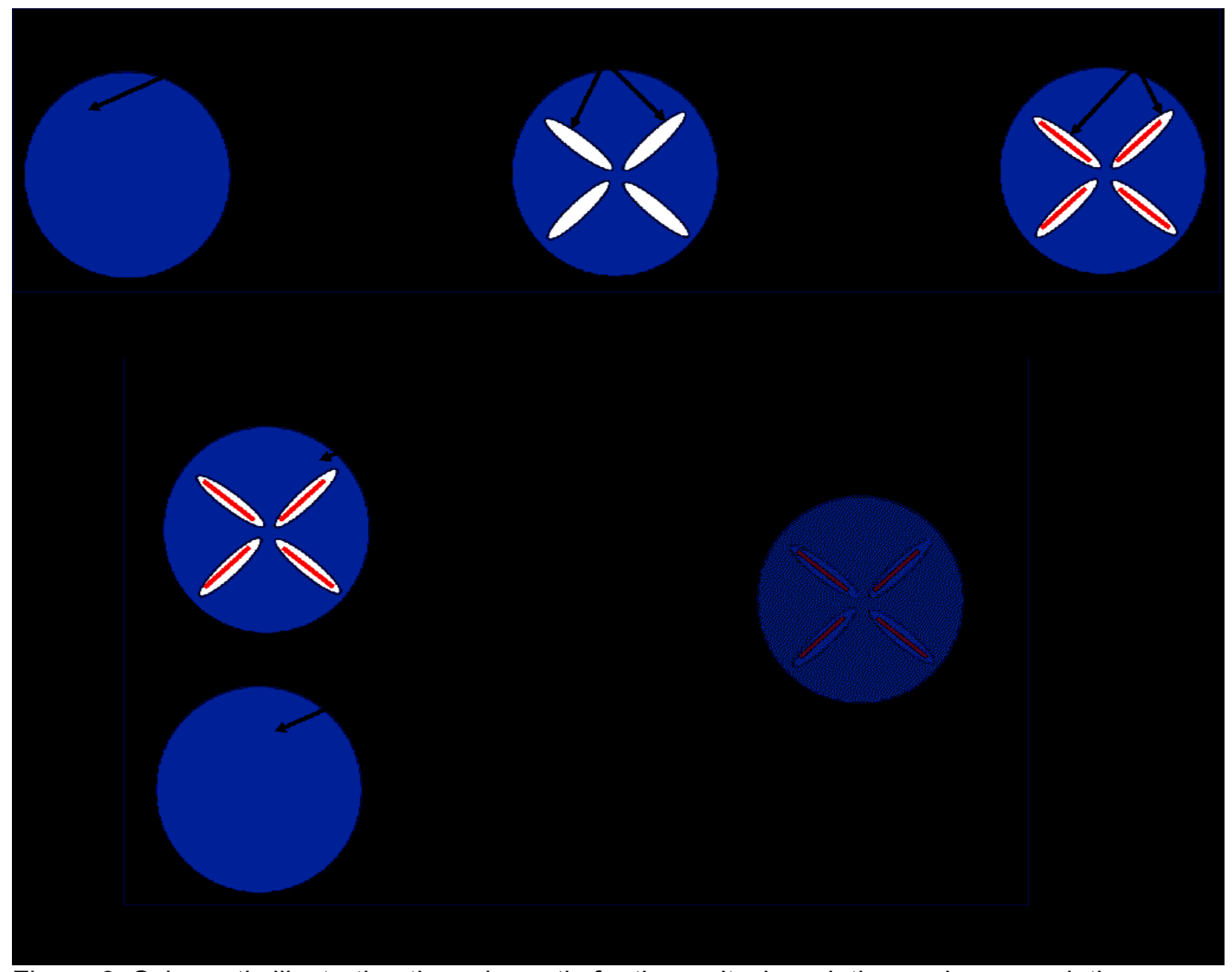

Figure 3. Schematic illustrating the schematic for the melt wire printing and encapsulation. 


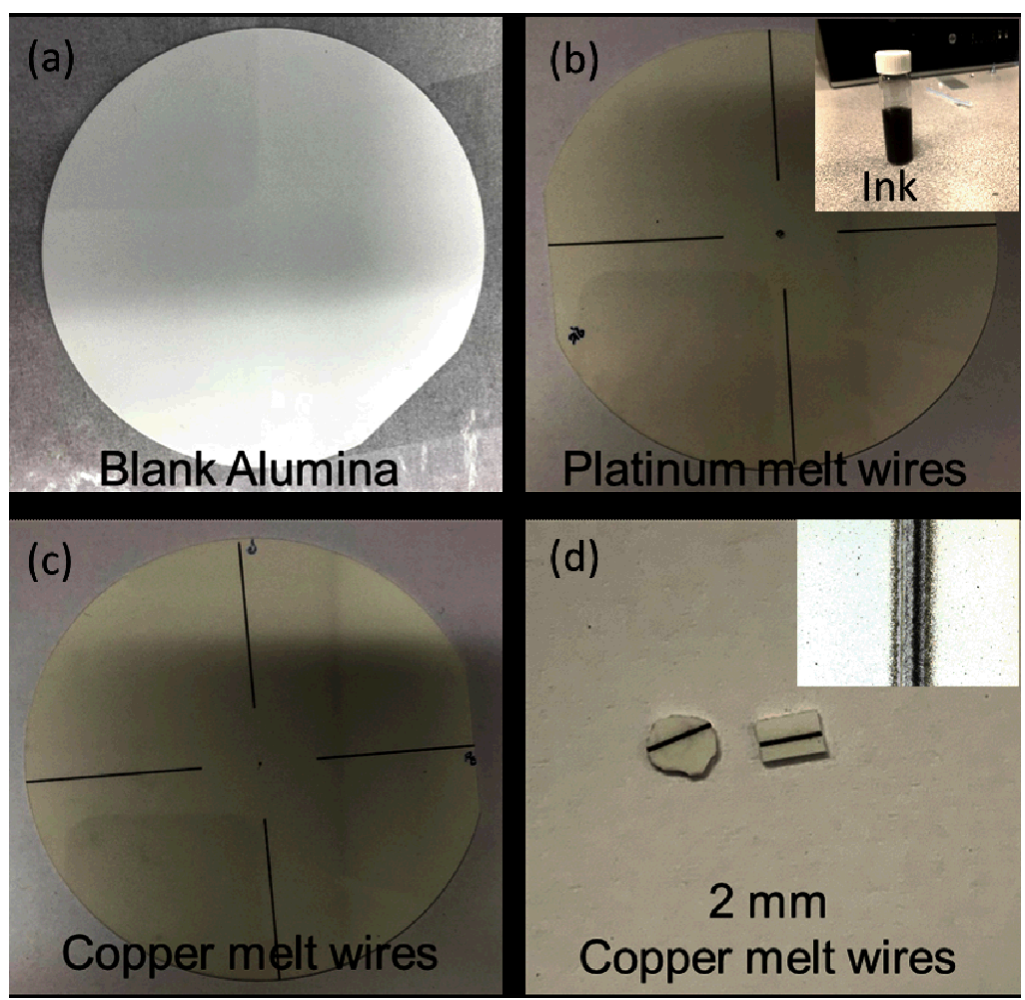

Figure 4. Printing of melt wires. (a) A blank alumina wafer as a model test substrate, (b) printed melt wires with platinum nanoparticle ink. The inset showing the stock platinum ink solution prior to printing. (c) copper nanoparticle ink as melt wires, and (d) micromachined copper melt wires to fit in the $2 \mathrm{~mm}$-diameter test reactor modules. The inset shows the optical micrograph of the printed wire. 


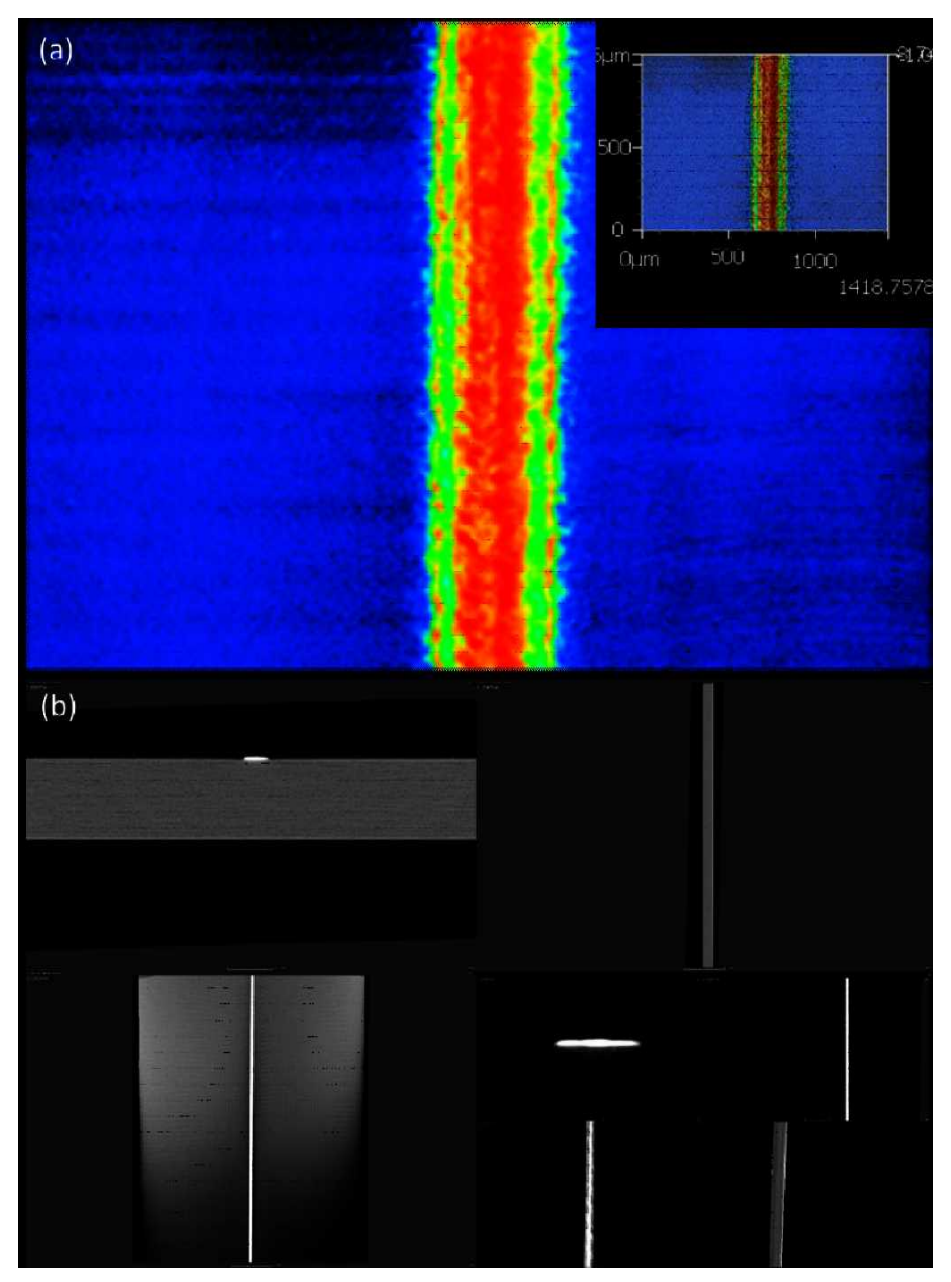

Figure 5. Characterization of printed melt wires. (a) Laser microscopic image of a copper melt wire. The inset shows 3D topography of the wire to estimate width and height of the wire. (b) Xray computer tomography (CT scan) of the melt wires to confirm that the line will be visible during and after exposure.

\section{Computational approach}

A modeling/simulation approach is being used to further understand the effects of processing parameters on the printed material. While plasma jet printing is of interest on the experimental side, the plasma interaction with the inks is poorly understood and difficult to model. The AJP process, which is still of interest to this program, is being modelled instead, which, while the sintering process is simplified, still will provide valuable processing information for the PJP process as well (e.g. effects of ink nano-particle sizes on the sintered film).

The AJP process can be broadly described as a two-step process. The first step is to deposit nanoparticles in a specified geometry, and the second step is to sinter the particles into a cohesive film. Sintering is one of the most regularly used additive manufacturing processes. The central obligation for the process to be "sintering" is that the particles are heated (sintered) to just below the melting point. This is achieved by regular thermal heating or by directing the laser pulse to heat the material. Sintering of nanoparticle is a big challenge in additive 
manufacturing printing. Sintering is compulsory for printing to cure the ink materials to reach its ultimate shape/morphology. For aerosol jet and plasma jet printings, sintering is essential to consolidate the micro/nano particles in the ink to achieve surface finish and to reach minimum resolution limit. Sometimes, sintering helps to modify phase morphology of materials in the ink. An external heating is must for aerosol jet printed patterns whereas, for plasma jet printing, plasma helps in sintering. Also, sintering plays an important role to adhere the printed pattern on the substrate. There is not much literature that exists which addressed the sintering of nanoparticle aiming the estimation of energy of surface adhesion.

This modeling effort is intended to address the sintering step by determining the best sintering conditions for multiple new inks, as well as mixed inks that have a mix of several compositions in the nano-particles. There are three features which are considered when determining which sintering parameters are best. The first feature is the microstructure of the sintered particles. In general, it is preferable to minimize void and defects, or at a minimum, be able to predict them. This will be addressed using phase field simulations. The second feature is the contact area between the substrate and the particles. This is important because there have been adhesion issues between the substrate and the printed films, as shown in Fig. 6. These problems may be mitigated by accounting for the contact area, the adhesion force per area, which may be computed from density functional theory (DFT), and the forces produced by the differing thermal expansions in the substrate and film. Phase field will be used to scale up the DFT results. The final feature to consider is the extent of diffusion between the particles. This is an important consideration when producing a specific alloy from mixed inks is necessary. DFT will be used to atomistically examine diffusion rates, and this will be scaled up in phase field.

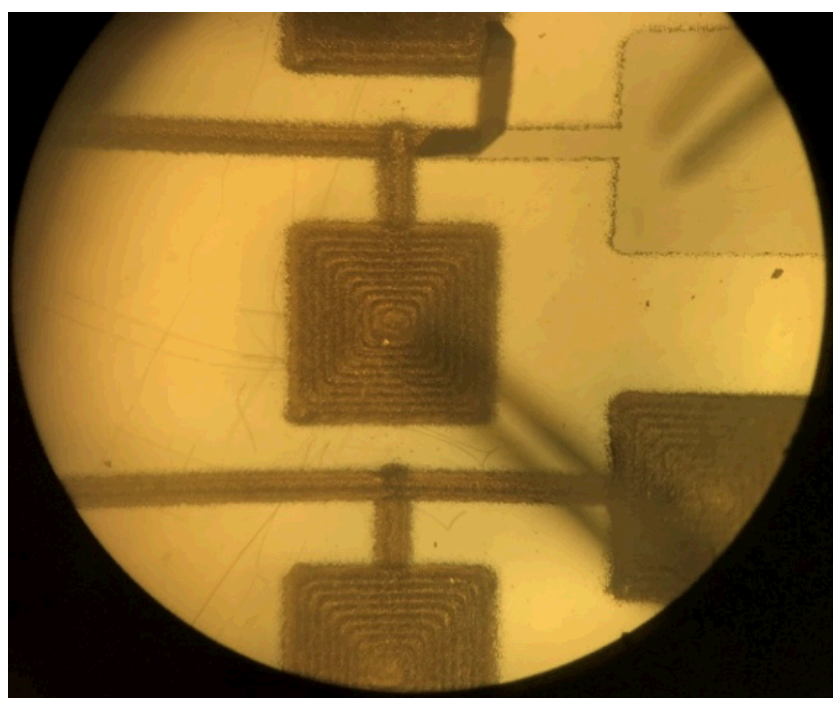

Figure 6. Detached printed Ni sensor

\subsection{Phase Field Modeling Overview}

A grand-potential based phase field model is used to simulate sintering of the metallic nanoparticles on an alumina substrate. This phase-filed formulation is presented in Aagesen et al.[6] and is implemented in the MOOSE framework. The particle diameters in the following simulations were set at $20 \mathrm{~nm}$ which is within the 10 to $100 \mathrm{~nm}$ range seen in transmission electron microscopic (TEM) images like Fig. 7. 


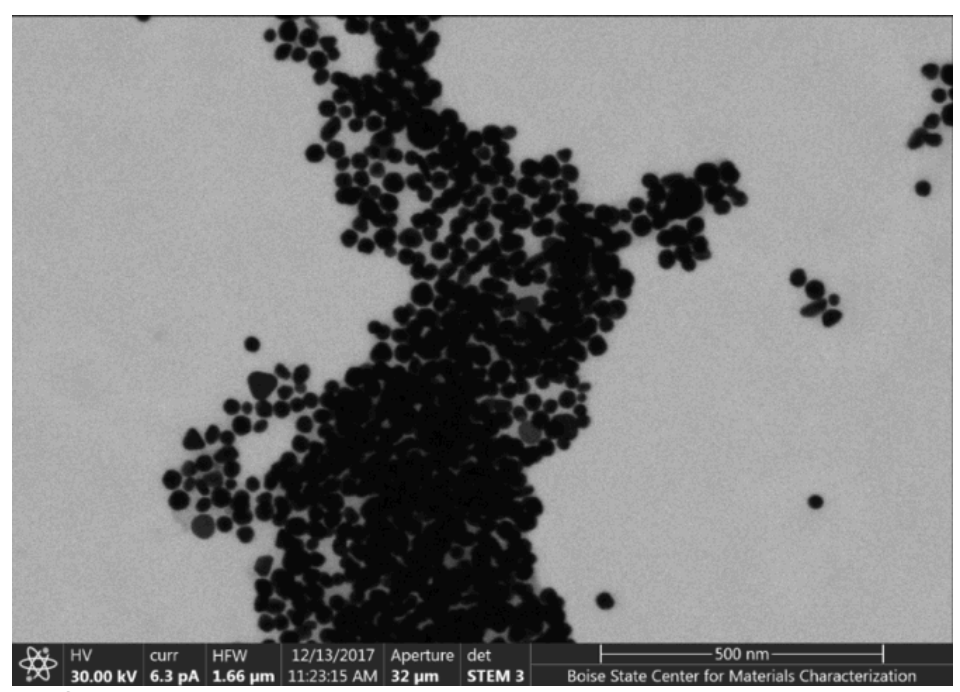

Figure 7. TEM Image of nanoparticles

The first simulations completed in this work are intended to verify the formulation and ensure the physics which occurs during sintering is appropriately captured by the model. To do this, a 2D simulation is run which contains a single circular particle positioned above the substrate. In this simulation there are three phases, one for the nanoparticle, one for the substrate, and one for the vapor which is above the particle and substrate. The initial concentrations, which range from zero to one, are shown for each of the phases in the left column of Fig. 8. During the simulation, the systems equilibrates, and the particle wet the surface of the substrate. These equilibrated states for each of the phases are shown in the right column of Fig. 8. It is worth noting that the parameters are set such that the substrate phase has minimal change while the particle and vapor deform around it.

A single particle starting geometry, like the one in Fig. 8, affords the ability to easily predict the final equilibrium geometry based on the input interfacial energies. Young's equation, equation 1, can be applied to compute the expected contact angle between the substrate and particle.

Here, $\theta$ is the angle between the substrate and the particle, are the surface energies between phases $x$ and $y$, and the subscripts represent particle, substrate, and vapor phases respectively. The angle computed with Young's equation can be compared to the simulation's equilibrium state as means of verification. Applying the surface energies from the simulation in Figure 3, Young's equation computes a contact angle of $90^{\circ}$, and from the right angle drawn in Figure $3 b$, this is shown to match the simulated results. Note that all simulations use the scale bar displayed on the right side of Fig. 8. 

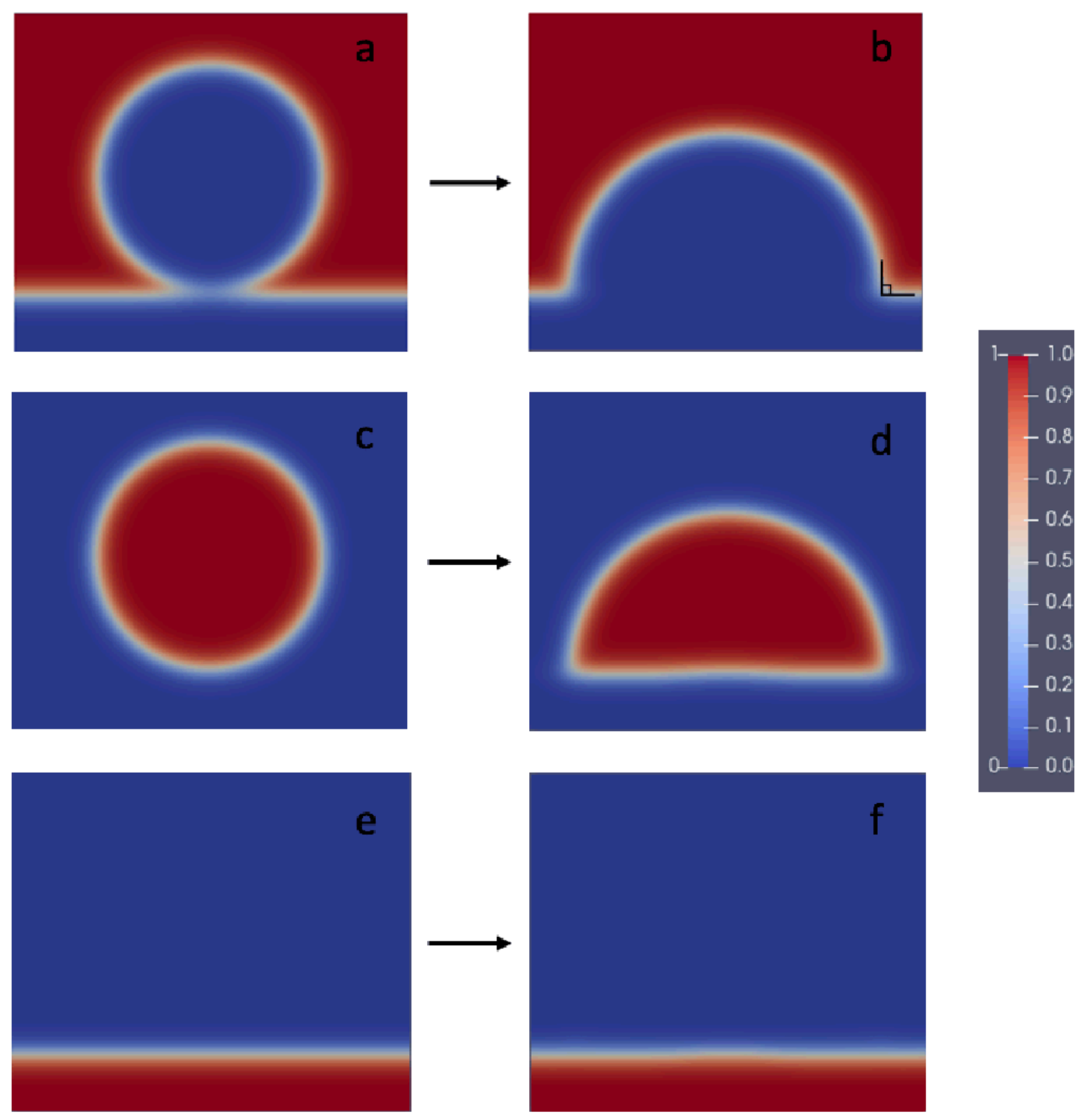

Figure 8. (a) Initial vapor phase concentration, (b) equilibrium vapor phase concentration, (c) Initial particle phase concentration, (d) equilibrium particle phase concentration, (e) Initial substrate phase concentration, (f) equilibrium substrate phase concentration.

\subsection{Material Specific Simulations}

\subsubsection{Single Particle Systems}

The parameters implemented in the simulation shown in Fig. 9 were chosen for easy verification. To produce results for the aerosol jet inks, several material specific parameters are necessary. These parameters are associated with the free energy, atomic diffusion, phase mobilities, and interfacial energies. Interfacial energies between alumina and several metals of 
interest are available in the literature and can be directly applied in the phase-field model. Fig. 9 $\mathbf{a}$ and $\mathbf{b}$ show the initial and equilibrium states of the vapor phase for a nickel nanoparticle on alumina at $1000{ }^{\circ} \mathrm{C}$ using interfacial energy values taken form Pilliar et al.[7] When the nickel system interfacial energies are used in equation 1 , it is found that simulation reproduces the expected contact angle.
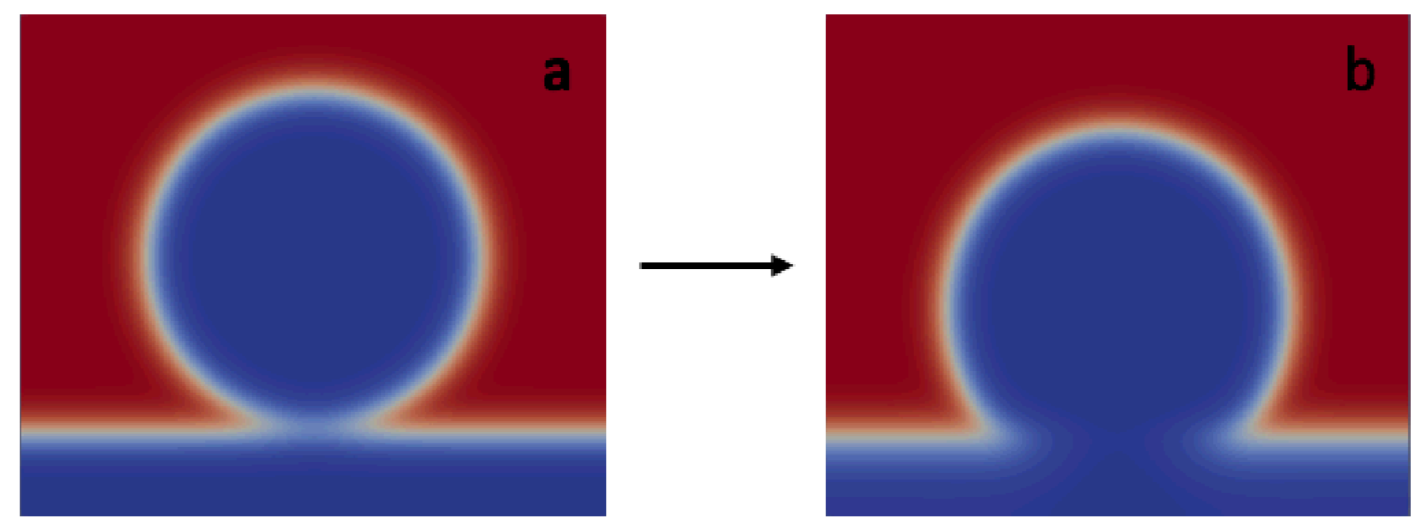

Figure 9. Initial (a) and equilibrium (b) vapor phase concentration for simulation of nickel on alumina substrate at $1000^{\circ} \mathrm{C}$.

\subsubsection{Multi-Particle Simulations}

Promising results have been produced for simulations containing two particles sintering. Fig. 10 and Fig. 11 show simulations for similar and dissimilar metal particles respectively. The results from the multiparticle simulations produce results with the expected behavior.
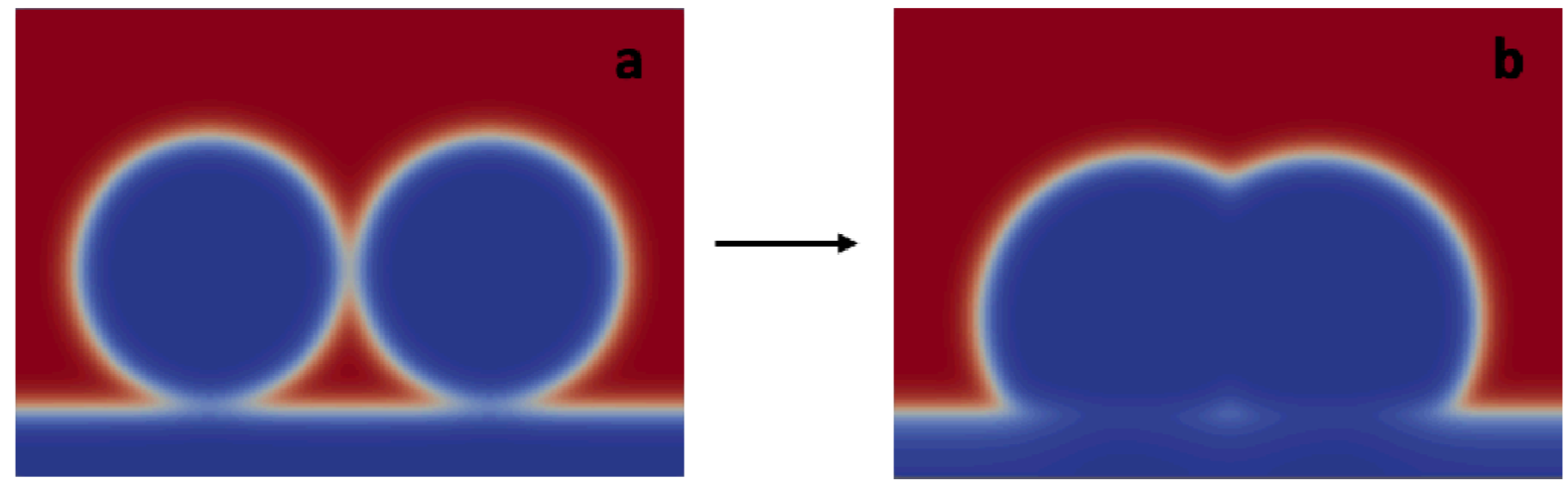

Figure 10. Initial(a) and equilibrium(b) vapor phase concentration for sintered nickel particles on alumina substrate at $1000^{\circ} \mathrm{C}$. 


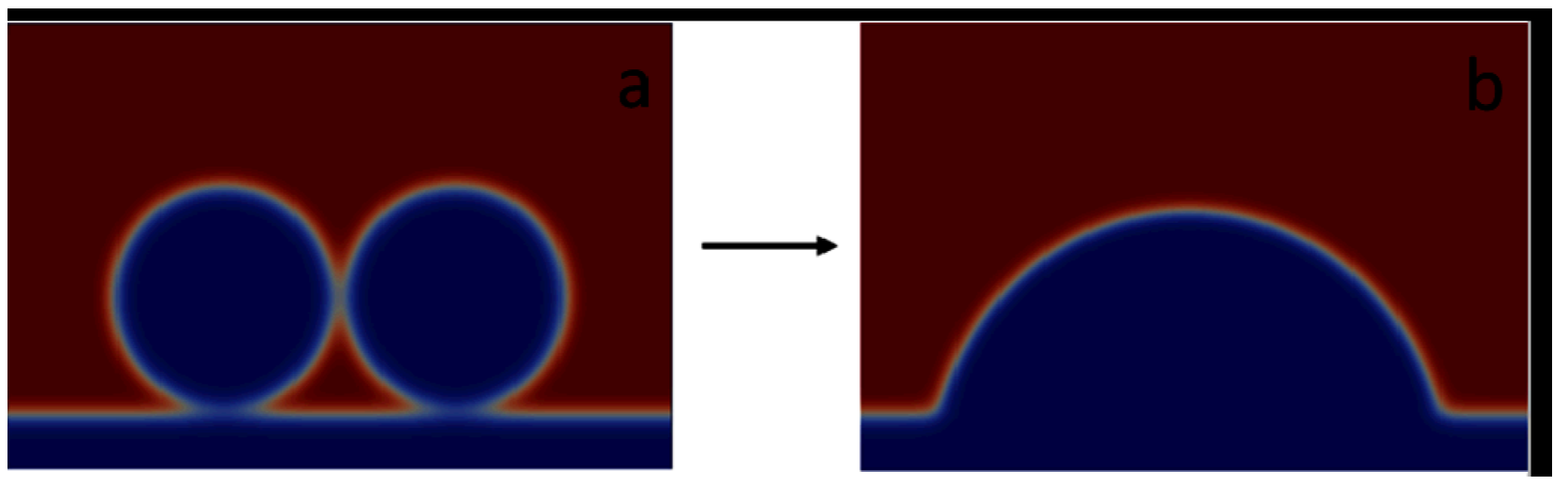

Figure 11. Initial (a) and equilibrium (b) vapor phase concentration for sintered $\mathrm{Al}(\mathrm{left})$ and $\mathrm{Ag}$ (right) particles on alumina.

\section{Nanoparticle (NP) sintering: A density functional theory (DFT) approach}

Here, our idea was to model the sintering behavior of nanoparticles at elevated temperatures using density functional theory (DFT). In previously work, molecular dynamics (MD) was used to examine this process created a simulation that incorporated metal nanoparticles and a substrate. However, due to limitations of resources and strict time frame with the potentials that describe the atomic interactions, the substrate-particle potential was modeled as a Lennard-Jones type interaction rather than something more realistic. The same issue arises when looking at different materials other than the copper and silver which were modeled. This was one of the main motivations for attempting to use DFT to model sintering DFT doesn't require specific potentials for each unique system.

Shown in Fig. 12 is a simulation of two $1 \mathrm{~nm}$ diameter nanoparticles merging together using ab-initio molecular dynamics (a combination of DFT and MD methods that allows for temperature control). As can be seen, the two nanoparticles neck at around $500 \mathrm{~K}$ and merge at around $800 \mathrm{~K}$. The main issue with this model is its scale - it is too small to get any good information out of it and cannot be feasibly scaled up any more without significant performance/calculation time issues. This was the main motivation for moving to phase-fields methods, so that the DFT results could be used as input into phase field and the phase field could model larger, more realistic size scales. 


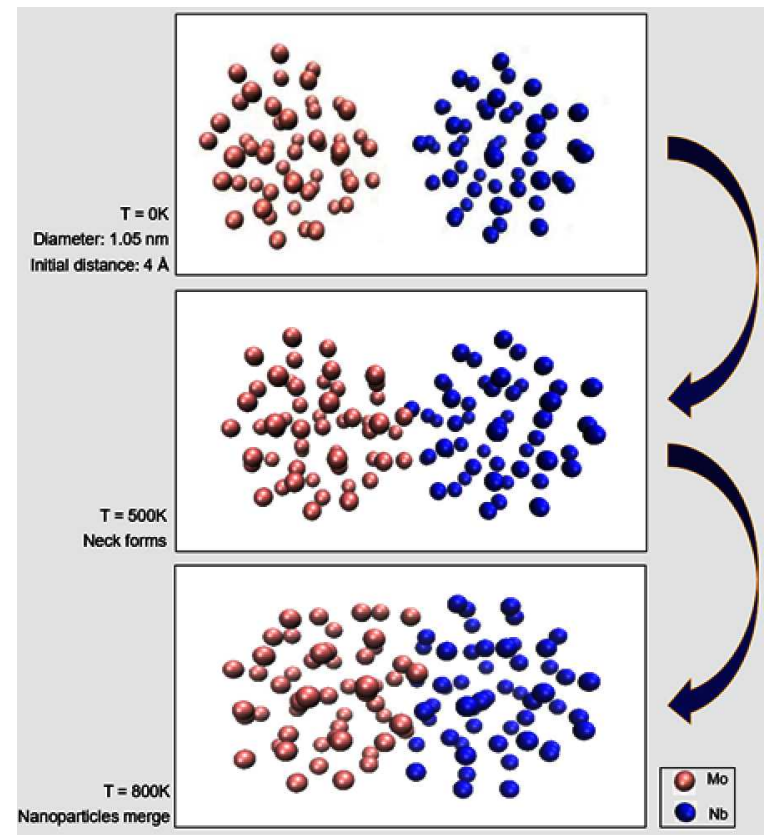

Figure 12. Sintering of two $1 \mathrm{~nm}$ nanoparticles (one Mo and one $\mathrm{Nb}$ ) using ab-initio molecular dynamics from 0K-800K.

The phase-field model requires the input of free surface and interfacial energies, both of which can be found using DFT, although for the systems was used to test the model this summer these values already exist in literature. To make sure that we can find the values in the future, however, we replicated a study done on an Al surface slab. The study, done by SinghMiller and Marzari,[8] reported the free surface energy of an Al slab terminated at the (001) surface. To replicate this study, we made 15 slabs with varying surface areas and atomic layers and found the surface energy of each slab using the equation

$(2)$

where is the number of unit cells and is the area of the slab, shown in Fig. 13a. Shown in Fig. $13 \mathrm{~b}$ is a slab with 6 atomic layers, where the plots of surface energy vs. number of atomic layers leveled out. Shown in Table 1 are the literature surface energies and my calculated surface energy.

(a) Surface Energy vs. Number Atomic Layers for Al Slab

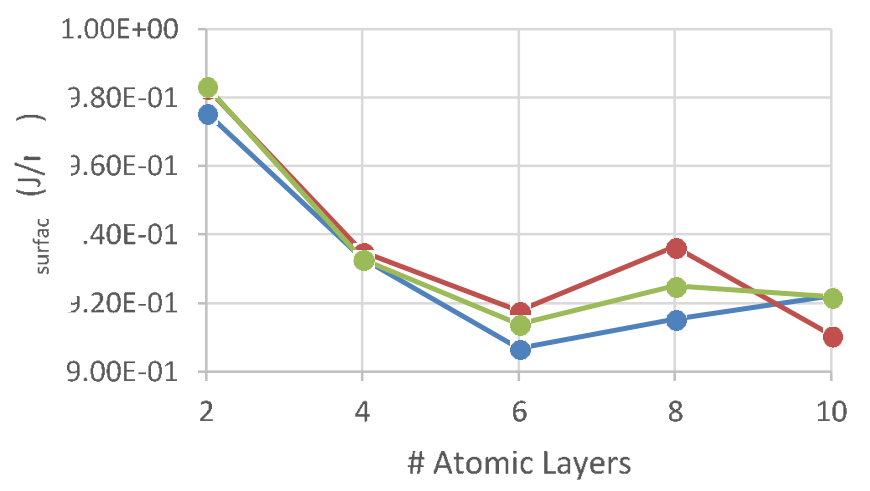

(b)

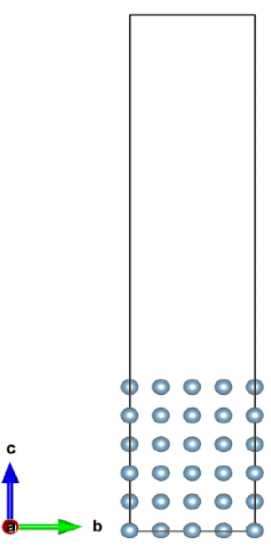


Figure 13. (a) Plots of surface energy vs. number of atomic layers for various Al slabs of different sizes. (b) Al slab with 6 atomic layers.

Table 1. Literature value for Al surface energy compared to my calculations. [8]

\begin{tabular}{|l|l|}
\hline DFT - LDA & $\gamma_{\text {surface }}\left(\mathrm{J} / \mathrm{m}^{2}\right)=0.91$ \\
\hline Expt. & $\gamma_{\text {surface }}\left(\mathrm{J} / \mathrm{m}^{2}\right)=1.14$ \\
\hline Replication & $Y_{\text {surface }}\left(\mathrm{J} / \mathrm{m}^{2}\right)=0.93$ \\
\hline
\end{tabular}

Once systems are identified for which we are unable to find published values for (or for which we want to use our own calculations), we can use this method to find free surface energies.

Similar to the free surface energy calculations, interfacial calculations will be done on systems to get interfacial energies. The benefits will be two-fold: getting interfacial energies and adhesion information using density of states and works of separation per area. The work of separation, found using the equation[9]

can be combined with the phase-field model from which the area of wetting by the nanoparticle can be found and multiplied by the work of separation per area to get a quantitative adhesion value. Currently, no work has been done with this since we didn't have access to the required interface-building software this summer at Idaho National Laboreatory (INL), but the process is well established and should be straightforward once the systems are made. Shown in Fig. 14 is a rudimentary interface of Mo and alumina made without the necessary software that contains bad structural errors.

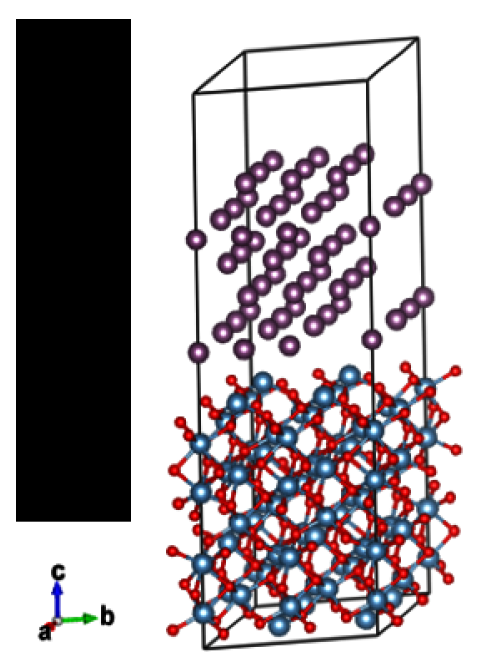

Figure 14. Interface of Mo and Alumina made without necessary interface-building software.

\section{Conclusions}

The initial studies on printings show promising results in printing nanoparticle inks. Copper a platinum melt wires were printed so far on alumina surface. The minimum resolution achieved 
by plasma jet printer was $146 \mu \mathrm{m}$ in case of copper ink. The $\mathrm{x}$-ray CT scans shows excellent imaging of melt wires which will be backbone of characterization for the melt wire at next stage during radiation tests. Our results highlight our ability to use AM techniques such as PJP and AJP to print melt wires and neutron dosimeters. Apart from experimental approach, a portion of the effort has been directed towards computational approach to address better process control. There are two primary sintering conditions that can be influenced by this work, sintering temperature and sintering time. In order to produce meaningful results which can be used to adjust these conditions, the simulation input parameters must be time and temperature dependent. At this time, we have only run simulations using parameters found in the literature and order of magnitude approximations. This is limiting because the literature does not necessarily have values for the desired materials or temperatures, and the order of magnitude approximations increase uncertainty. Future work will include obtaining parameters from CALPHAD, complementary DFT work at BSU, and other sources in order to produce more welltuned results and provide optimal conditions for the experimental work. 


\section{References}

[1] R.P. Gandhiraman, E. Singh, D.C. Diaz-Cartagena, D. Nordlund, J. Koehne, M. Meyyappan, Plasma jet printing for flexible substrates, Appl. Phys. Lett. 108 (2016) 123103. doi:10.1063/1.4943792.

[2] R.P. Gandhiraman, V. Jayan, J.-W. Han, B. Chen, J.E. Koehne, M. Meyyappan, Plasma Jet Printing of Electronic Materials on Flexible and Nonconformal Objects, ACS Appl. Mater. Interfaces. 6 (2014) 20860-20867. doi:10.1021/am505325y.

[3] A. Dey, S. Krishnamurthy, J. Bowen, D. Nordlund, M. Meyyappan, R.P. Gandhiraman, Plasma Jet Printing and in Situ Reduction of Highly Acidic Graphene Oxide, ACS Nano. 12 (2018) 5473-5481. doi:10.1021/acsnano.8b00903.

[4] M. Tsumaki, K. Nitta, S. Jeon, K. Terashima, T. Ito, Development of plasma-assisted inkjet printing and demonstration for direct printing of conductive silver line, J. Phys. Appl. Phys. 51 (2018) 30LT01. doi:10.1088/1361-6463/aac7db.

[5] ANTISTATIC AGENT INCORPORATION METHOD AND ITS PERFORMANCE, in: Handb. Antistatics, Elsevier, 2016: pp. 129-139. doi:10.1016/B978-1-895198-95-9.50012-1.

[6] L.K. Aagesen, Y. Gao, D. Schwen, K. Ahmed, Grand-potential-based phase-field model for multiple phases, grains, and chemical components, Phys. Rev. E. 98 (2018) 023309. doi:10.1103/PhysRevE.98.023309.

[7] R.M. Pilliar, J. Nutting, Solid-solid interfacial energy determinations in metal-ceramic systems, Philos. Mag. 16 (1967) 181-188. doi:10.1080/14786436708229267.

[8] N.E. Singh-Miller, N. Marzari, Surface energies, work functions, and surface relaxations of low-index metallic surfaces from first principles, Phys. Rev. B. 80 (2009) 235407. doi:10.1103/PhysRevB.80.235407.

[9] X. Li, Q. Hui, D. Shao, J. Chen, P. Wang, Z. Jia, C. Li, Z. Chen, N. Cheng, First-principles study on the stability and electronic structure of Mg/ZrB2 interfaces, Sci. China Mater. 59 (2016) 28-37. doi:10.1007/s40843-016-0118-x. 